\title{
TESTICULAR ARTERY ARISING FROM AN ABERRANT RIGHT RENAL ARTERY
}

\author{
Suluba HE, Otieno E \\ Correspondence to Dr. Emmanuel Henry Suluba, Department of Anatomy, School of Medicine, \\ Muhimbili University of Health and Allied Sciences (MUHAS), P.O Box 65001, Dar-es-Salaam,Tanzania. \\ E-mail: emmasulubahenry@yahoo.com, Mobile: +255 754936150
}

\section{ABSTRACT}

Testicular arteries usually arise from the abdominal Aorta; however they may rarely arise from other arteries of posterior abdominal wall. Variations of the testicular arteries and renal vessels are common. Awareness of these variations is very important to surgeons to increase their surgical precision and therefore avoiding iatrogenic injuries as well as useful in diagnostic procedures. This case report we discovered the rare variation of the origin of the right testicular artery arising from the right aberrant renal artery with double renal artery irrigating both left and right kidneys. These variations in the testicular arteries and renal arteries have implication to surgical procedures such as orchidopexy repair for undescended testis, renal transplantation and nephrectomy. Thus with the introduction of novel surgical and invasive diagnostic procedures understanding of these rare variations becomes significantly important. Currently these procedures are of increasing in our settings as such the information on variations is of prime importance.

Keywords: Testicular artery; renal artery, variation, abdominal aorta.

\section{INTRODUCTION}

The testicular arteries usually originate from the abdominal aorta after the origin of the renal arteries at the level of the second lumbar vertebra(Singh, 2011).It courses inferolaterally inclose proximity with psoas major on its way to the pelvis. On the right side the testicular artery it is related anteriorly on its course to the inferior vena cava, posteriorly to the branches of superior mesenteric artery. On the left side courses posterior to the inferior mesenteric vein, left colic artery and the descending colon. Both right and left testicular arteries as they enter the pelvis, they lie anterior to the genitofemoral nerves, ureters and external iliac arteries. Both arteries then enter the deep internal inguinal ring and travel with the ipsilateral spermatic cord in the inguinal canal to the scrotum.(Susan,2016).

Many studies studies (Bandopadhyay \& Saha,2009;Satheesha Nayak B,2008;Rusu,2006;Sontakke,Tarnekar and
Bokariya 2013) have described the variations of anatomy of the vessels such as testicular arteries, ovarian arteries, renal arteries and mesenteric arteries originating from the abdominal aorta. Embryologically the vasculature of kidneys and gonads are derived from the lateral mesonephric branches of dorsal aorta(Dey,2016). Knowledge on variations origin of testicular artery and veins are very important during performance of surgical procedures. The accidental damage of these vessels may lead to the damage of the testis. The gonadal artery may pass posterior near to the ureter that can interrupt in its pathway leading to hydronephrosis.(Wadhwa and Soni,2010) With recently increase of the number of abdominal surgical interventions and radiological investigations a good knowledge of the anatomy of gonadal arteries is of prime importance. In this case we describe the variation of testicular artery and renal artery found on the male cadaver during routine 
abdominal wall.

\section{CASE REPORT}

This case report describes the variation of the testicular artery. The variation was found during routine dissection of the structures of the posterior abdominal wall. We open the abdomen and removal of the visceral organs, which are found within the peritoneum. The retroperitoneal structures could therefore be studied. We found double renal arteries supplied the right kidney, the right testicular artery originate from aberrant of the renal artery. The left testicular artery was classical arising from the abdominal aorta. Both kidneys were normal in terms of size and position. No any other malformation was noted.

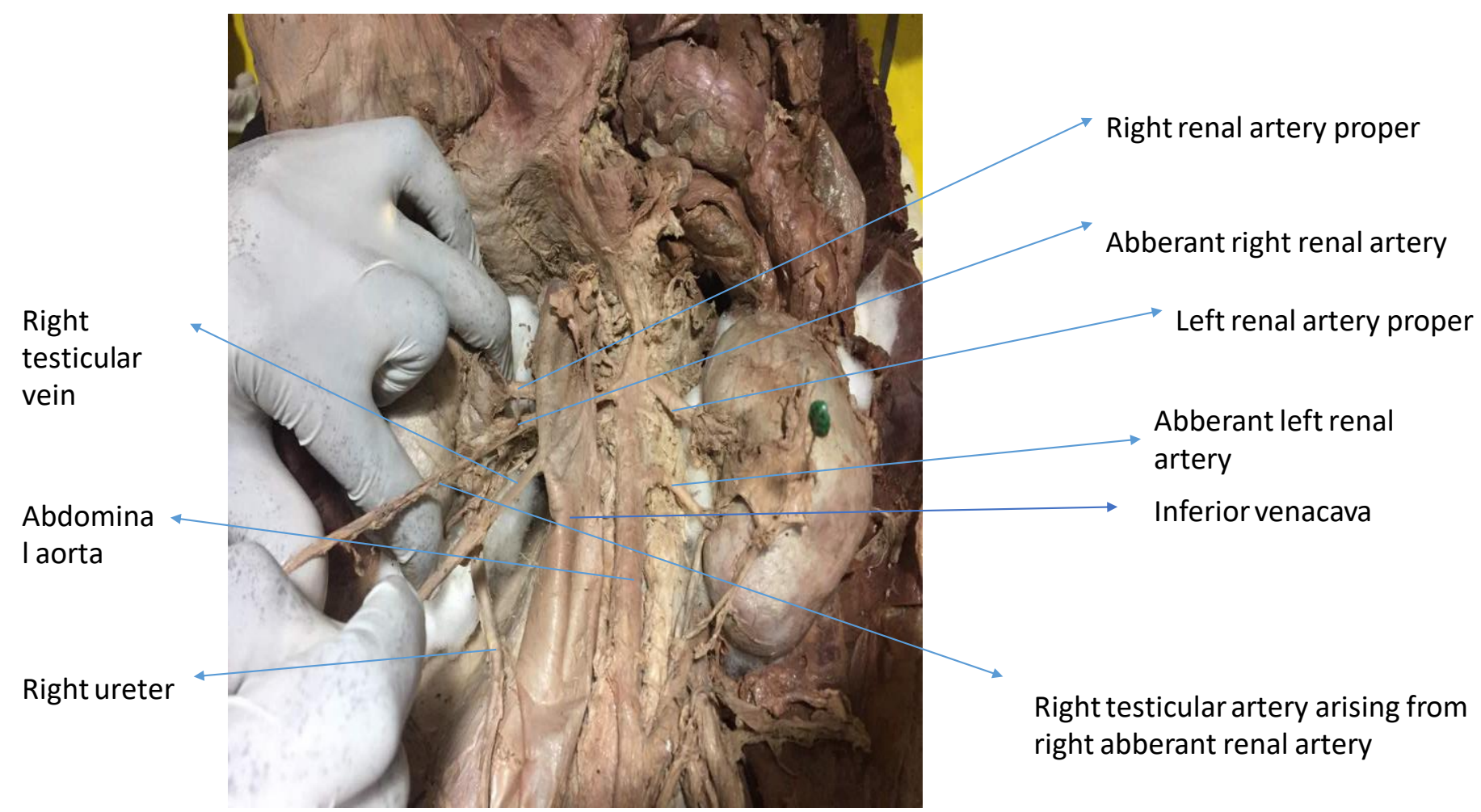

\section{DISCUSSION}

Variations of the origin of the vessels arising from the abdominal aorta are common. Classically both the right and left testicular arteries, which supplies the testis, arises from the abdominal aorta. However many variations about testicular artery can occur. The abnormal course of the left testicular artery was discovered during routine dissection of the posterior abdominal wall, in the same study two renal arteries arose from the abdominal aorta at a distance of about $2 \mathrm{~cm}$ from each other on the left side (Ranade, 2007).

Another study among people of European origin the double renal and testicular arteries arising from the right superior renal artery were found(Rusu, 2006).The rare variations of the course of the gonadal arteries has been described in other different 
studies(Bandopadhyay \& Saha,2009;Satheesha,2008;Rahman,Dong and Yamadori,1993). In another study the left testicular artery arises from superior polar renal artery from the left side and the right kidney were supplied by the triple renal arteries all originating from the abdominal aorta(Rai,2013). The bilateral testicular artery were also arising from the aberrant renal artery in India cadaveric study, in similar study the kidneys were supplied by double renal arteries(Savithri,2014) .The study done among 42 cadavers in Indian population shows about $4(10 \%)$ were arising from renal artery(Sushma R.Kotian,2016).An unusual case was found in 55 year old man where the right testicular artery arises from the right accessory artery whereas on the left side the left testicular arteries were double both arising from the accessory renal arteries(Sontakke, Tarnekar, and Bokariya ,2013). In our case the left testicular artery arises from the left aberrant renal artery this is different from the so far reported studies. These discussed anatomical variations are clinically important in performing surgical procedures such as nephrectomy, since the ligation of the renal artery may accidentally cut the blood supply to the testis leading to testicular atrophy in case of the above unnoticed variations.

In conclusion, during diagnostic and surgical approaches at the levels of the renal, gonadal and hepatic vessels, not only the single or unilateral anatomical variants must be considered, but also associations of these and the bilateral presence must be suspected and it is clear that in order to avoid any iatrogenic damage to important structures and their complications, a great knowledge on the variation of these vessels it shouldn't be overlooked.

\section{REFERENCES}

1. Bandopadhyay, Manimay, and Anubha Saha. 2009. "Three Rare Variations in the Course of the Gonadal Artery." 27(3): 655-58.

2. Dey, Saikat Kumar, Pallab Kumar Saha, Anupam Baske, and Parijat Mukherjee. 2016. "Variation of Origin of Left Testicular Artery- A Case Report." (June): 377-81.

3. Rahman, H. A., K. Dong, and T. Yamadori. 1993. "Unique Course of the Ovarian Artery Associated with Other Variations." Journal of Anatomy 182(Pt 2): 287.

4. Rai, Rajalakshmi, Anu V Ranade, Latha V Prabhu, and Ashwin R Rai. 2013. "Variant Left Testicular Artery from the Superior Polar Artery and Triple Right Renal Arteries : A Case Report." 17(4): 262-64.

5. Ranade, Anu V,Rai R,Pruhbu L V,Mangala K,Nayak S R, 2007. "Arched Left Gonadal Artery over the Left Renal Vein Associated with Double Left Renal Artery." Singapore Med J48(12): 332-34.

6. Rusu, M. C. 2006. "Human Bilateral Doubled Renal and Testicular Arteries with a Left Testicular Arterial Arch around the Left Renal Vein." Rom J Morphol Embryol = Revue roumaine de morphologie et embryologie 47(2): 197-200.

7. Satheesha Nayak B.2008 Case Report,. "Abnormal Course of Left Ovarian Artery." : 4-5.

8. Savithri P.2014"Bilateral variation in the origin of testicular artery from renal artery-case report of a rare variant"National journal of clinical Anatomy vol-3(1):32-36

9. Singh,R;Jaiswal,A;Shamal,S.N and Singh S.P. 2011. "Variation in the Origin of the Testicular Arteries and Drainage of the Right Testicular Vein." 29(2): 614-16.

10. Sontakke, B R, A M Tarnekar, and P Bokariya. 2013. "An Unusual Case Of Asymmetrical \& V ariant Origin Of Gonadal Arteries In A Male Cadaver." 18(March): 61-63.

11. Susan Standring. 2016.Gray's Anatomy:Anatomical basis of clinical practice. 41th Ed.London:Elsevier Limited, p1272.

12. Sushma R.Kotian,Arnhd Kumar Pandey,Supriya Padmashali,Judith Jaison,Sneha Guruprasad Kaltheu,2016. "Original article A Cadaveric Study of the Testicular Artery and Its Clinical 
Significance." 15(4): 280-86.

13. Wadhwa, Ambica, and Sandeep Soni. 2010. "Clinical Medicine Insights: Reproductive Health A Study of Gonadal Arteries in 30 Adult Human Cadavers." Clinical Medicine Insights: Reproductive Health 4: 1-5. 